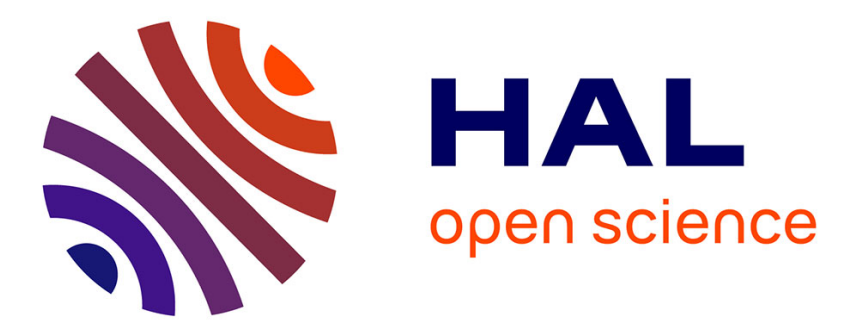

\title{
Co-scheduling algorithms for cache-partitioned systems
}

Guillaume Aupy, Anne Benoit, Loïc Pottier, Padma Raghavan, Yves Robert, Manu Shantharam

\section{To cite this version:}

Guillaume Aupy, Anne Benoit, Loïc Pottier, Padma Raghavan, Yves Robert, et al.. Co-scheduling algorithms for cache-partitioned systems. APDCM 2017 - 19th Workshop on Advances in Parallel and Distributed Computational Models, May 2017, Orlando (FL), United States. pp.1-10, 10.1109/IPDPSW.2017.60 . hal-01654660

\section{HAL Id: hal-01654660 \\ https://hal.inria.fr/hal-01654660}

Submitted on 4 Dec 2017

HAL is a multi-disciplinary open access archive for the deposit and dissemination of scientific research documents, whether they are published or not. The documents may come from teaching and research institutions in France or abroad, or from public or private research centers.
L'archive ouverte pluridisciplinaire HAL, est destinée au dépôt et à la diffusion de documents scientifiques de niveau recherche, publiés ou non, émanant des établissements d'enseignement et de recherche français ou étrangers, des laboratoires publics ou privés. 


\title{
Co-scheduling algorithms for cache-partitioned systems
}

\author{
Guillaume Aupy*, Anne Benoit ${ }^{\dagger}$, Loïc Pottier $^{\dagger}$, Padma Raghavan*, Yves Robert ${ }^{\dagger \ddagger}$, Manu Shantharam ${ }^{\S}$ \\ *Vanderbilt University, Nashville TN, USA \\ ${ }^{\dagger}$ Laboratoire LIP, École Normale Supérieure de Lyon, France \\ $\ddagger$ University of Tennessee, Knoxville TN, USA \\ ${ }^{\S}$ San Diego Supercomputer Center, San Diego CA, USA
}

\begin{abstract}
Cache-partitioned architectures allow subsections of the shared last-level cache (LLC) to be exclusively reserved for some applications. This technique dramatically limits interactions between applications that are concurrently executing on a multicore machine. Consider $n$ applications that execute concurrently, with the objective to minimize the makespan, defined as the maximum completion time of the $n$ applications. Key scheduling questions are: (i) which proportion of cache and (ii) how many processors should be given to each application? Here, we assign rational numbers of processors to each application, since they can be shared across applications through multi-threading. In this paper, we provide answers to (i) and (ii) for perfectly parallel applications. Even though the problem is shown to be NP-complete, we give key elements to determine the subset of applications that should share the LLC (while remaining ones only use their smaller private cache). Building upon these results, we design efficient heuristics for general applications. Extensive simulations demonstrate the usefulness of co-scheduling when our efficient cache partitioning strategies are deployed.
\end{abstract}

\section{INTRODUCTION}

At scale, the I/O movements of HPC applications are expected to be one of the most critical problems [1]. Observations on the Intrepid machine at Argonne National Laboratory (ANL) show that I/O transfers can be slowed down up to $70 \%$ due to congestion [10]. When ANL upgraded its house supercomputer from Intrepid (Peak perf: 0.56 PFlops; peak I/O throughput: $88 \mathrm{~GB} / \mathrm{s}$ ) to Mira (Peak perf: 10 PFlops; peak I/O throughput: $240 \mathrm{~GB} / \mathrm{s}$ ), the net result for an application whose I/O throughput scales linearly (or worse) with performance was a downgrade from $160 \mathrm{~GB} / \mathrm{PFlop}$ to $24 \mathrm{~GB} / \mathrm{PFlop!}$

To cope with such an imbalance (which is not expected to reduce on future platforms), a possible approach is to develop in situ co-scheduling analysis and data preprocessing on dedicated nodes [1]. This scheme applies to data-intensive periodic workflows where data is generated by the main simulation, and parallel processes are run to process this data with the constraints that output results should be sent to disk storage before newly generated data arrives for processing. These solutions are starting to be implemented for HPC applications. Sewell et al. [27] explain that in the case of the HACC application (a cosmological code), petabytes of data are created to be analyzed later. The analysis is done by multiple independent processes. The idea of their work is to minimize the amount of data copied to I/O filesystem, by performing the analysis at the same time as HACC is running (what they call in situ). The main constraint is that these processes are data-intensive and are handled by a dedicated machine. Also, the execution of these processes should be done efficiently enough so that they finish before the next batch of data arrives, hence resulting in a pipelined approach. All these frameworks motivate the design of efficient co-scheduling strategies.

One main issue of co-scheduling is to evaluate co-run degradations due to cache sharing [31]. Many studies have shown that interferences on the shared last-level cache (LLC) can be detrimental to co-scheduled applications [20]. Previous solutions consisted in preventing co-schedule of possibly interfering workloads, or terminating low importance applications [29]. Lo et al. [21] recently showed experimentally that important gains could be reached by co-scheduling applications with strict cache partitioning enabled. Cache partitioning, the technique at the core of this work, consists in reserving exclusivity of subsections of the LLC of a chip multi-processor (CMP), to some of the applications running on this CMP. This functionality was recently introduced by Intel under the name Cache Allocation Technology [15]. With the advent of large shared memory multi-core machines (e.g., Sunway TaihuLight, the current \#1 supercomputer uses 256-cores processor chips with a shared memory of 32GB [8]), the design of algorithms that co-schedule applications efficiently and decide how to partition the shared memory (seen as the cache here), is becoming critical.

In this work, we study the following problem: given a set of parallel applications, a multi-core processor with a shared last-level cache LLC, how can we best partition the LLC to minimize the total execution time (or makespan), i.e., the moment when the last application finishes its computation. For each application, we assume that we know the number of compute operations to perform, and the miss rate on a fixed size cache. For the multi-core processor, we know its LLC size, the cost for a cache miss, the cost for a cache hit, the size of the cache and total number of processors. We assume that these processors can be shared by two applications through multi-threading [17], hence we assign fractional number of processors to each application. In addition to being very natural in practice, this relaxation of the problem avoids artefacts due to rounding when assigning only integer numbers of processors, and allows us to study the intrinsic complexity of co-scheduling with cache partitioning. Equipped with all 
these applications and platform parameters, recent work [13], [26], [17] shows how to model the impact of cache misses and to accurately predict the execution time of an application. In this context, we make the following main contributions:

- We show that the co-scheduling problem is NP-complete, even when applications are perfectly parallel, i.e., their speedup scales up linearly with the number of processors.

- In the case of perfectly parallel applications, we show several results that characterize optimal solutions. We show that the co-scheduling cache-partitioning problem reduces to deciding which subset of applications will share the LLC; when this subset is known, we show how to determine the optimal cache fractions and number of processors.

- While these results only hold for perfectly parallel applications, they guide the design of heuristics for general applications. We show through extensive simulations that our heuristics greatly improve the performance of cachepartitioning algorithms, even for parallel applications obeying Amdahl's law with a large sequential fraction, hence with a limited speedup profile.

The rest of the paper is organized as follows. Section II provides an overview of related work. Section III is devoted to formally defining the framework and all model parameters. Section IV gives our main theoretical contributions. The heuristics are defined in Section $\mathrm{V}$, and evaluated through simulations in Section VI. Finally, Section VII outlines our main findings and discusses directions for future work.

\section{RELATED WORK}

Since the advent of systems with tens of cores, coscheduling has received considerable attention. Due to lack of space, we refer to [23], [7], [21] for a survey of many approaches to co-scheduling. The main idea is to execute several applications concurrently rather than in sequence, with the objective to increase platform throughput. Indeed, some individual applications may well not need all available cores, or some others could use all resources, but at the price of a dramatic performance loss. In particular, the latter case is encountered whenever application speedup becomes too low beyond a given processor count.

The main difficulty of co-scheduling is to decide which applications to execute concurrently, and how many cores to assign to each of them. Indeed, when executing simultaneously, any two applications will compete for shared resources, which will create interferences and decrease their throughput. Modeling application interference is a challenging task. Dynamic schedulers are used when application behavior is unknown [25], [28]. Static schedulers aim at optimizing the sharing of the resources by relying on application knowledge such as estimated workload, speed-up profile, cache behavior, etc. One widely-used approach is to build an interference graph whose vertices are applications and whose edges represent degradation factors [16], [30], [14]. This approach is interesting but hard to implement. Indeed, the interaction of two applications depends on many factors, such as their size, their core count, the memory bandwidth, etc. Obtaining the speedup profile of a single application already is difficult and requires intensive benchmarking campaigns. Obtaining the degradation profile of two applications is even more difficult and can be achieved only for regular applications. To further darken the picture, the interference graph subsumes only pairwise interactions, while a global picture of the processor and cache requirements for all applications is needed by the scheduler.

Shared resources include cache, memory, I/O channels and network links, but among potential degradation factors, cache accesses are prominent. When several applications share the cache, they are granted a fraction of cache lines as opposed to the whole cache, and their cache miss ratio increases accordingly. Multiple cache partitioning strategies have been proposed [6], [12], [5], [9]. In this paper, we focus on a static allocation of LLC cache fractions, and processor numbers, to concurrent applications as a function of several parameters (cache-miss ratio, access frequency, operation count). To the best of our knowledge, this work is the first analytical model and complexity study for this challenging problem.

\section{MODEL}

This section details all platform and application parameters, and formally states the optimization problem.

Architecture. We consider a parallel platform of $p$ homogeneous computing elements, or processors, that share two storage locations:

- A small storage $\mathcal{S}_{s}$ with low latency, governed by a LRU replacement policy, also called cache;

- A large storage $\mathcal{S}_{l}$ with high latency, also called memory. More specifically, $C_{s}$ (resp. $C_{l}$ ) denotes the size of $\mathcal{S}_{s}$ (resp. $\left.\mathcal{S}_{l}\right)$, and $l_{s}$ (resp. $l_{l}$ ) the latency of $\mathcal{S}_{s}\left(\right.$ resp. $\left.\mathcal{S}_{l}\right)$. In this work, we assume that $C_{l}=+\infty$. We have the relation $l_{s} \ll l_{l}$.

In this work, we consider the cache partitioning technique [15], where one can allocate a portion of the cache to applications so that they can execute without interference from other applications.

Applications. There are $n$ independent parallel applications to be scheduled on the parallel platform, whose speedup profiles obey Amdahl's law [2]. For an application $T_{i}$, we define several parameters:

- $w_{i}$, the number of computing operations needed for $T_{i}$;

- $s_{i}$, the sequential fraction of $T_{i}$;

- $f_{i}$, the frequency of data accesses of $T_{i}: f_{i}$ is the number of data accesses per computing operation;

- $a_{i}$, the memory footprint of $T_{i}$.

We use these parameters to model the execution of each application as follows.

a) The power law of cache misses: In chip multiprocessors, many authors have observed that the Power Law accurately models how the cache size affects the miss rate [13], [26], [17]. Mathematically, the power law states that if $m_{0}$ is the miss rate of a workload for a baseline cache size $C_{0}$, the miss rate $m$ for a new cache size $C$ can be expressed as $m=m_{0}\left(\frac{C_{0}}{C}\right)^{\alpha}$ where $\alpha$ is the sensitivity factor from the 
Power Law of Cache Misses [13], [26], [17] and typically ranges between 0.3 and 0.7 with an average at 0.5 . Note that, by definition, a rate cannot be higher than 1 , hence we extend this definition as:

$$
m=\min \left(1, m_{0}\left(\frac{C_{0}}{C}\right)^{\alpha}\right) .
$$

This formula can be read as follows: if the cache size allocated is too small, then the execution goes as if no cache was allocated, and all accesses will be misses.

b) Computations and data movement: We use the cost model introduced by Krishna et al. [17] to evaluate the execution cost of an application as a function of the cache fraction that it has been allocated. Specifically, for each application, we define $m_{0}$, the miss rate of application $T_{i}$ with a cache of size $C_{0}$ (we can also use the miss rate of applications with a cache of another fixed size). We express the execution time of $T_{i}$ as a function of $p_{i}$, the number of processors allocated to $T_{i}$, and $x_{i}$, the fraction of $\mathcal{S}_{s}$ allocated to $T_{i}$ (recall both are rational numbers). Let $F l_{i}\left(p_{i}\right)$ be the number of operations performed by each processor for application $T_{i}$, given that the application is executed on $p_{i}$ processors. We have $F l_{i}\left(p_{i}\right)=s_{i} w_{i}+\left(1-s_{i}\right) \frac{w_{i}}{p_{i}}$ according to Amdahl's speedup profile. Finally,

$$
\mathcal{E x e}_{i}\left(p_{i}, x_{i}\right)= \begin{cases}F l_{i}\left(p_{i}\right)\left(1+f_{i}\left(l_{s}+l_{l}\right)\right) & \text { if } x_{i}=0 ; \\ F l_{i}\left(p_{i}\right)\left(1+f_{i}\left(l_{s}+l_{l} \cdot \min \left(1, \frac{m_{0}}{\left(\frac{x_{i} C_{s}}{C_{0}}\right)^{\alpha}}\right)\right)\right) & \quad \text { if } x_{i} C_{s} \leq a_{i} ; \\ F l_{i}\left(p_{i}\right)\left(1+f_{i}\left(l_{s}+l_{l} \cdot \min \left(1, \frac{m_{0}}{\left(\frac{a_{i}}{C_{0}}\right)^{\alpha}}\right)\right)\right) & \text { otherwise. }\end{cases}
$$

Indeed, for each operation, we pay the cost of the computing operation, plus the cost of data accesses, and by definition we have $f_{i}$ accesses per operation. At each access, we pay a latency $l_{s}$, and an additional latency $l_{l}$ in case of cache miss (see Equation (1)). The last case states that we cannot use a portion of cache greater than the memory footprint $a_{i}$ of application $T_{i}$. This model is somewhat pessimistic: cache accesses to the same variable by two different processors are counted twice. We show in Section VI that despite this conservative assumption (no sharing), co-scheduling can outperform classical approaches that sequentially deploy each application on the whole set of available resources.

Equation (2) calls for a few observations. For notational convenience, let $d_{i}=m_{0}\left(\frac{C_{0}}{C_{s}}\right)^{\alpha}$ :

- It is useless to give a fraction of cache larger than $\frac{a_{i}}{C_{s}}$ to application $T_{i}$;

- Because of the minimum $\min \left(1, \frac{d_{i}}{\left(x_{i}\right)^{\alpha}}\right)$, either $x_{i}>d_{i}^{\frac{1}{\alpha}}$, or $x_{i}=0$ : indeed, if we give application $T_{i}$ a fraction of cache smaller than $d_{i}^{\frac{1}{\alpha}}$, the minimum is equal to 1 , and this fraction is wasted.

Hence, we have for all $i$ :

$$
x_{i}=0 \text { or } d_{i}^{\frac{1}{\alpha}}<x_{i} \leq \frac{a_{i}}{C_{s}} .
$$

Of course, if $d_{i}^{\frac{1}{\alpha}} \geq \frac{a_{i}}{C_{s}}$ for some application $T_{i}$, then $x_{i}=0$.

Scheduling problem. Given $n$ applications $T_{1}, \ldots, T_{n}$, we aim at partitioning the shared cache and assign processors so that the concurrent execution of these applications takes minimal time. In other words, we aim at minimizing the execution time of the longest application, when all applications start their execution at the same time. Formally:

Definition 1 (COSCHEDCACHE). Given $n$ applications $T_{1}, \ldots, T_{n}$ and a platform with $p$ identical processors sharing a cache of size $C_{s}$, find a schedule $\left\{\left(p_{1}, x_{1}\right), \ldots,\left(p_{n}, x_{n}\right)\right\}$ with $\sum_{i=1}^{n} p_{i} \leq p$, and $\sum_{i=1}^{n} x_{i} \leq 1$, that minimizes

$$
\max _{1 \leq i \leq n} \mathcal{E} e_{i}\left(p_{i}, x_{i}\right) \text {. }
$$

\section{Complexity Results for Perfectly Parallel APPLICATIONS}

In this section, we consider COSCHEDCACHE with perfectly parallel applications. A perfectly parallel application $T_{i}$ is an application with $s_{i}=0$, so that $\mathcal{E x e}_{i}\left(p_{i}, x_{i}\right)=\frac{\mathcal{E x e}_{i}\left(1, x_{i}\right)}{p_{i}}$. Let $\mathcal{E x e}_{i}^{\text {seq }}\left(x_{i}\right)=\mathcal{E x e}_{i}\left(1, x_{i}\right)$ be the sequential execution time of application $T_{i}$ with a fraction of cache $x_{i}$. The main results are the NP-completeness of COSCHEDCACHE, and several dominance results on the optimal solution. While these results only hold for perfectly parallel applications, they will guide the design of heuristics for general applications in Section V.

\section{A. Intractability}

We formally state the decision problem associated to COSCHEdCACHE:

Definition 2 (COSCHEDCACHE-DEC). Given $n$ perfectly parallel applications $T_{1}, \ldots, T_{n}$ and a platform with $p$ identical processors sharing a cache of size $C_{s}$, and given a bound $K$ on the makespan, does there exist a schedule $\left\{\left(p_{1}, x_{1}\right), \ldots,\left(p_{n}, x_{n}\right)\right\}$, where $p_{i}$ and $x_{i}$ are nonnegative rational numbers with $\sum_{i=1}^{n} p_{i} \leq p$ and $\sum_{i=1}^{n} x_{i} \leq 1$, such that $\max _{1 \leq i \leq n} \mathcal{E x e}_{i}\left(p_{i}, x_{i}\right) \leq K$ ?

\section{Theorem 1. CoSchedCACHE-DeC is NP-complete.}

Proof. For perfectly parallel applications, we can transform CoSchedCache into an equivalent problem that does not depend on the number of processors but that relies simply on the cache partitioning strategy (Lemma 3 below). This result will guide processor assignment for general applications in Section V. We start with a few lemmas. The first lemma shows that all applications complete at the same time in an optimal execution:

Lemma 1. To minimize the makespan, all applications must finish at the same time.

Proof. Consider the $n$ perfectly parallel applications $T_{1}, \ldots, T_{n}$ and a solution $\mathcal{S}=\left\{\left(p_{i}, x_{i}\right)\right\}_{1 \leq i \leq n}$ to CoSchedCACHE. Let $D_{\mathcal{S}}=\max _{i} \mathcal{E x e}_{i}\left(p_{i}, x_{i}\right)$ be the makespan of this solution. We denote by $I_{\mathcal{S}}$ the set of applications whose execution time is exactly $D_{\mathcal{S}}$. 
We show the result by contradiction. We consider an optimal solution $\mathcal{S}$ whose subset $I_{\mathcal{S}}$ has minimal size (i.e., for any other optimal solution $\mathcal{S}_{o},\left|I_{\mathcal{S}}\right| \leq\left|I_{\mathcal{S}_{o}}\right|$ ). Then we show that if $\left|I_{\mathcal{S}}\right| \neq n$, we can construct a solution $\mathcal{S}^{\prime}$ with either (i) a smaller makespan if $\left|I_{\mathcal{S}}\right|=1$ (contradicting the optimality hypothesis), or (ii) one less application whose execution time is exactly $D_{\mathcal{S}}$ (contradicting the minimality hypothesis). Assume $\left|I_{\mathcal{S}}\right| \neq n$, let $T_{i_{0}} \notin I_{\mathcal{S}}$ and $T_{i_{1}} \in I_{\mathcal{S}}$. We have $\mathcal{E X e}_{i_{0}}\left(p_{i_{0}}, x_{i_{0}}\right)<\mathcal{E} \mathrm{Xe}_{i_{1}}\left(p_{i_{1}}, x_{i_{1}}\right)=D_{\mathcal{S}}$. We define

$$
\begin{aligned}
& \varepsilon=p_{i_{0}} p_{i_{1}} \frac{\mathcal{E x e}_{i_{1}}\left(p_{i_{1}}, x_{i_{1}}\right)-\mathcal{E} \mathrm{Xe}_{i_{0}}\left(p_{i_{0}}, x_{i_{0}}\right)}{\mathcal{E} \mathrm{xe}_{i_{0}}^{\mathrm{seq}^{2}}\left(x_{i_{0}}\right)+\mathcal{E} \mathrm{Xe}_{i_{1}}^{\mathrm{seq}_{1}}\left(x_{i_{1}}\right)} \\
& =\frac{p_{i_{0}} \mathcal{E x e}_{i_{1}}^{\mathrm{seq}}\left(x_{i_{1}}\right)-p_{i_{1}} \mathcal{E x e}_{i_{0}}^{\mathrm{seq}}\left(x_{i_{0}}\right)}{\mathcal{E x e}_{i_{0}}^{\mathrm{seq}}\left(x_{i_{0}}\right)+\mathcal{E} \mathrm{xe}_{i_{1}}^{\mathrm{seq}}\left(x_{i_{1}}\right)}>0 .
\end{aligned}
$$

Then clearly, $\mathcal{S}^{\prime}=\left\{\left(p_{i}^{\prime}, x_{i}\right)\right\}_{i}$ where $p_{i}^{\prime}$ is (i) $p_{i}$ if $i \notin\left\{i_{0}, i_{1}\right\}$, (ii) $p_{i_{0}}-\varepsilon$ if $i=i_{0}$, (iii) $p_{i_{1}}+\varepsilon$ if $i=i_{1}$, is a valid solution: we have the property $\sum_{i} p_{i}^{\prime}=\sum_{i} p_{i} \leq p$, and $\sum_{i} x_{i}^{\prime}=\sum_{i} x_{i} \leq 1$.

Furthermore, one can verify that $\mathcal{E x e}_{i_{1}}\left(p_{i_{1}}^{\prime}, x_{i_{1}}\right)=$ $\mathcal{E} \mathrm{xe}_{i_{0}}\left(p_{i_{0}}^{\prime}, x_{i_{0}}\right)=\frac{\mathcal{E x e}_{i_{0}}^{\mathrm{seq}}\left(x_{i_{0}}\right)+\mathcal{E} \mathrm{xe}_{i_{1}}^{\mathrm{seq}}\left(x_{i_{1}}\right)}{p_{i_{0}}+p_{i_{1}}}$. Because $p_{i_{1}}^{\prime}>p_{i_{1}}$, $\mathcal{E x e}_{i_{1}}\left(p_{i_{1}}^{\prime}, x_{i_{1}}\right)<\mathcal{E}_{\mathrm{Xe}_{i_{1}}}\left(p_{i_{1}}, x_{i_{1}}\right)=D_{\mathcal{S}}$. Hence:

- If $\left|I_{\mathcal{S}}\right|=1$, then for all $i, \mathcal{E x e}_{i}\left(p_{i}^{\prime}, x_{i}\right)<D_{\mathcal{S}}$, hence showing that $\mathcal{S}$ is not optimal.

- Else, $I_{\mathcal{S}^{\prime}}=I_{\mathcal{S}^{\prime}} \backslash\left\{i_{1}\right\}$, and $D_{\mathcal{S}^{\prime}}=D_{\mathcal{S}}$, hence showing that $\mathcal{S}$ is not minimal.

This shows that necessarily, $\left|I_{\mathcal{S}}\right|=n$.

The second lemma shows the optimal processor assignment:

Lemma 2. Given $n$ applications $T_{1}, \ldots, T_{n}$ and a partitioning of the cache $\left\{x_{1}, \ldots, x_{n}\right\}$, then the optimal number of processors for application $T_{i}(i \in\{1, \ldots, n\})$ is:

$$
p_{i}=p \frac{\mathcal{E} x e_{i}^{s e q}\left(x_{i}\right)}{\sum_{j=1}^{n} \mathcal{E} x e_{j}^{s e q}\left(x_{j}\right)} .
$$

Proof. According to Lemma 1, all applications finish at the same time. Given $i_{0} \in\{1, \ldots, n\}$, we have $\frac{\mathcal{E x e}_{i_{0}}^{\text {seq }}\left(x_{i_{0}}\right)}{p_{i_{0}}}=$ $\frac{\mathcal{E x e}_{i}^{\text {seq }}\left(x_{i}\right)}{p_{i}}$ for all $1 \leq i \leq n$. In addition, we have $\sum_{i=1}^{n} p_{i}=p$ : the fact that this bound is tight in an optimal solution is due to the fact that we have perfectly parallel applications. We express $p$ in terms of the others variables, and we do the summation: $p=\sum_{i=1}^{n} p_{i}=\frac{p_{i_{0}}}{\mathcal{E x e}_{i_{0}}^{\mathrm{sec}}\left(x_{i_{0}}\right)} \sum_{i=1}^{n} \mathcal{E} \mathrm{xe}_{i}^{\mathrm{seq}}\left(x_{i}\right)$. This directly leads to the result.

Lemmas 1 and 2 lead to the following reformulation of COSCHEDCACHE:

Lemma 3. COSCHEDCACHE can be rewritten as finding the optimal cache partitioning strategy $\mathcal{X}=\left\{x_{1}, \ldots, x_{n}\right\}$ that minimizes the completion time of an optimal solution:

$$
\frac{1}{p} \sum_{i=1}^{n} \mathcal{E} x e_{i}\left(1, x_{i}\right)
$$

Proof. Lemma 2 gives us that in an optimal solution the processor distribution is uniquely determined by the cache partitioning strategy. Furthermore, given a cache partitioning strategy, we know that all applications finish at the same time (Lemma 1) and that the completion time is equal to

$$
\frac{\mathcal{E x e}_{1}^{\text {seq }}\left(x_{1}\right)}{p_{1}}=\frac{\sum_{i=1}^{n} \mathcal{E} \mathrm{xe}_{i}^{\mathrm{seq}}\left(x_{i}\right)}{p} .
$$

We are now ready for the proof of Theorem 1 . COSCHEDCACHE-DEC is obviously in NP: given the $x_{i}$ 's, it is easy to verify all constraints in linear time. We prove the completeness by a reduction from KNAPSACK, which is NP-complete [11]. Consider an arbitrary instance $\mathcal{I}_{1}$ of KNAPSACK: given $n$ objects, each with positive integer size $u_{i}$ and positive integer value $v_{i}$ for $1 \leq i \leq n$, and two positive integer bounds $U$ and $V$, does there exist a subset $I \subset\{1, \ldots, n\}$ such that $\sum_{i \in I} u_{i} \leq U$ and $\sum_{i \in I} v_{i} \geq V$ ? Given $\mathcal{I}_{1}$, we construct the following instance $\mathcal{I}_{2}$ of COSCHEDCACHE-DEC: - We define two constants $\varepsilon=\frac{1}{N(N+1)}$ and $\eta=1-\frac{1}{N}$, where $N=\max (n, 2 U+1)$.

- We let $d_{i}=\left(\frac{u_{i} \eta}{U}\right)^{\alpha}, e_{i}=\left(d_{i}^{\frac{1}{\alpha}}+\varepsilon\right)^{\alpha}, a_{i}=e_{i}^{\frac{1}{\alpha}} C_{s}$, and $w_{i} f_{i} l_{l}=\frac{v_{i}}{1-\frac{d_{i}}{e_{i}}}$ for $1 \leq i \leq n$. Note that we only need the value of the product $w_{i} f_{i}$, and we can set one of them arbitrarily.

- The bound $K$ is defined as:

$$
p K=\sum_{i=1}^{n} w_{i}\left(1+f_{i} l_{s}\right)+\sum_{i=1}^{n} w_{i} f_{i} l_{l}-V .
$$

To simplify notations, let $z_{i}=w_{i} f_{i} l_{l}$. Letting $A=$ $\sum_{i=1}^{n} w_{i}\left(1+f_{i} l_{s}\right)$ and $Z=\sum_{i=1}^{n} z_{i}$, we get $p K=A+Z-V$. Also, we have $\sum_{i=1}^{n} w_{i}\left(1+f_{i}\left[l_{s}+l_{l} \cdot \min \left(1, \frac{d_{i}}{x_{i}^{\alpha}}\right)\right]\right)=$ $A+B$, where $B=\sum_{i=1}^{n} z_{i} \min \left(1, \frac{d_{i}}{x^{\alpha}}\right)$. Recall from Lemma 3 that $\mathcal{I}_{2}$ has a solution if and only if $\frac{1}{p}(A+B) \leq K$.

Let $I_{C} \subseteq\{1, \ldots, n\}$ denote the subset of applications that are given some cache $\left(x_{i} \neq 0\right.$ if and only if $\left.i \in I_{C}\right)$. We also call $I_{C}$ the nonzero subset of $\mathcal{I}_{2}$. We have

$$
d_{i}^{\frac{1}{\alpha}} \leq x_{i} \leq \frac{a_{i}}{C_{s}}=e_{i}^{\frac{1}{\alpha}}
$$

so that we can rewrite $B=Z-\sum_{i \in I_{C}} z_{i}\left(1-\frac{d_{i}}{x_{i}^{\alpha}}\right)$. Given the value of the bound $K$, we have $A+B \leq p K$ if and only if

$$
\sum_{i \in I_{C}} z_{i}\left(1-\frac{d_{i}}{x_{i}^{\alpha}}\right) \geq V
$$

We show that $\mathcal{I}_{1}$ has a solution if and only if $\mathcal{I}_{2}$ does. Suppose first that $\mathcal{I}_{1}$ has a solution subset $I \subset\{1, \ldots, n\}$. Then we let $x_{i}=e_{i}^{\frac{1}{\alpha}}$ if $i \in I$ and $x_{i}=0$ otherwise. This is a valid solution to $\mathcal{I}_{2}$ with nonzero subset $I_{C}=I$. Indeed:

- If $i \in I$, then $d_{i}^{\frac{1}{\alpha}} \leq x_{i}=e_{i}^{\frac{1}{\alpha}}=\frac{a_{i}}{C_{s}}$.

- We have

$$
\sum_{i \in I} x_{i}=\sum_{i \in I}\left(d_{i}^{\frac{1}{\alpha}}+\varepsilon\right)=\sum_{i \in I} \frac{u_{i} \eta}{U}+|I| \varepsilon .
$$

But $\sum_{i \in I} \frac{u_{i} \eta}{U} \leq \eta$ (since we have a solution for $\mathcal{I}_{1}$ ), and $|I| \varepsilon \leq n \varepsilon \leq \frac{1}{N+1}$, hence $\sum_{i \in I} x_{i} \leq \eta+\frac{1}{N+1} \leq 1$. 
- Finally, $\sum_{i \in I} z_{i}\left(1-\frac{d_{i}}{x_{i}^{\alpha}}\right)=\sum_{i \in I} z_{i}\left(1-\frac{d_{i}}{e_{i}}\right)=\sum_{i \in I} v_{i} \geq$ $V$ (since we have a solution for $\mathcal{I}_{1}$ ), hence $A+B \leq p K$.

Suppose now that $\mathcal{I}_{2}$ has a solution, and let $I_{C}$ be its nonzero subset. We claim that $I=I_{C}$ is a solution to $\mathcal{I}_{1}$. Indeed, for $i \in I_{C}$ we have $d_{i} \leq x_{i}^{\alpha} \leq e_{i}$ and $\sum_{i \in I_{C}} z_{i}\left(1-\frac{d_{i}}{x_{i}^{\alpha}}\right) \geq V$. First, we have $\sum_{i \in I_{C}} z_{i}\left(1-\frac{d_{i}}{x_{i}^{\alpha}}\right) \geq$ $\sum_{i \in I_{C}} z_{i}\left(1-\frac{d_{i}^{i}}{e_{i}}\right)=\sum_{i \in I_{C}} v_{i}$, hence $\sum_{i \in I_{C}} v_{i} \geq V$. Then $\sum_{i \in I_{C}} d_{i}^{\frac{1}{\alpha}} \leq \sum_{i \in I_{C}} x_{i} \leq 1$, and $\sum_{i \in I_{C}} d_{i}^{\frac{1}{\alpha}}=\sum_{i \in I_{C}} \frac{u_{i} \eta}{U}$, hence $\sum_{i \in I_{C}} u_{i} \leq \frac{U}{\eta}$. But $\frac{U}{\eta} \leq U+\frac{1}{2}$ by the choice of $\eta$, thus $\sum_{i \in I_{C}} u_{i} \leq U+\frac{1}{2}$. Because the sizes are integers, $\sum_{i \in I_{C}} u_{i} \leq U$. Altogether, $I_{C}$ is indeed a solution to $\mathcal{I}_{1}$. This concludes the proof.

\section{B. Dominance results}

In this section, we provide dominance results that will guide the design of heuristics. In addition to restricting to perfectly parallel applications $\left(s_{i}=0\right)$, we assume that application memory footprints are larger than the cache size $\left(a_{i}=+\infty\right)$. The core of the previous intractability result relies on the hardness to determine the set of applications that receive a cache fraction (denoted by $I_{C}$ ) and those that do not (denoted by $\overline{I_{C}}$ ). In this section, we show (i) how to determine the optimal solution when these sets $I_{C}$ and $\overline{I_{C}}$ are known, and (ii) whether one can disqualify some partitions as being suboptimal.

In particular, we define a set of partitions of applications that we call dominant (Definition 4). We show that (i) if a partition of applications $I_{C}, \overline{I_{C}}$ is dominant, then we can compute the minimum execution time for this partition, and (ii) if a partition is not dominant, then we can find a better dominant partition. We start by rewriting the problem when the partitioning $I_{C}, \overline{I_{C}}$ of applications is known:

Definition 3 (COSCHEDCACHE-PART $\left(I_{C}, \overline{I_{C}}\right)$ ). Given a set of applications $T_{1}, \ldots, T_{n}$ and a partition $I_{C}, \overline{I_{C}}$, COSCHEDCACHE-PART $\left(I_{C}, \overline{I_{C}}\right)$ is the problem to find a set $\mathcal{X}=\left\{x_{1}, \ldots, x_{n}\right\}$ that minimizes the execution time:

$\frac{1}{p}\left(\sum_{i \in \overline{I_{C}}} w_{i}\left(1+f_{i}\left(l_{s}+l_{l}\right)\right)+\sum_{i \in I_{C}} w_{i}\left(1+f_{i} l_{s}+f_{i} l_{l} \frac{d_{i}}{x_{i}^{\alpha}}\right)\right)$

under the constraints $x_{i}=0$ if $i \in \overline{I_{C}}, x_{i}>d_{i}^{1 / \alpha}$ if $i \in I_{C}$, and $\sum_{1 \leq i \leq n} x_{i} \leq 1$.

We now relax some bounds in $\operatorname{CoSCHEDCACHE-PART}\left(I_{C}, \overline{I_{C}}\right)$ and define COSCHEDCACHE-EXT $\left(I_{C}, \overline{I_{C}}\right)$, which is the same problem except that the constraints on the $x_{i}$ 's when $i \in I_{C}$ is relaxed: we have instead $x_{i} \geq 0$ if $i \in I_{C}$.

A solution of CoSchedCACHE-PART $\left(I_{C}, \overline{I_{C}}\right)$ is a solution of COSCHEDCACHE-EXT $\left(I_{C}, \overline{I_{C}}\right)$, because we simply removed the constraints $x_{i}>d_{i}^{1 / \alpha}$ in the latter problem. Hence the execution time of the optimal solution of CoSchedCACHE-EXT $\left(I_{C}, \overline{I_{C}}\right)$ is lower than that of COSCHEDCACHE-PART $\left(I_{C}, \bar{I}_{C}\right)$. Furthermore, given a solution of CoSCHEDCACHE-EXT $\left(I_{C}, \overline{I_{C}}\right)$, one can easily see that its execution time in COSCHEDCACHE will be lower (the objective function is lower since it involves a minimum for all applications in $I_{C}$ ).

Lemma 4. Given a set of applications $T_{1}, \ldots, T_{n}$ and a partition $I_{C}, \overline{I_{C}}$, the optimal solution to COSCHEDCACHE-EXT $\left(I_{C}, \overline{I_{C}}\right)$ is

$$
\begin{array}{ll}
x_{i}=\frac{\left(w_{i} f_{i} d_{i}\right)^{1 /(\alpha+1)}}{\sum_{j \in I_{C}}\left(w_{j} f_{j} d_{j}\right)^{1 /(\alpha+1)}} & \text { if } i \in I_{C}, \\
x_{i}=0 & \text { otherwise. }
\end{array}
$$

Proof. We want to compute $\mathcal{X}=\left\{x_{1}, \ldots, x_{n}\right\}$ that minimizes the execution time. Discarding constant factors, this reduces to minimizing

$$
K(\mathcal{X})=\sum_{i \in I_{C}} \frac{w_{i} f_{i} d_{i}}{x_{i}^{\alpha}}
$$

under the constraints: $x_{i}=0$ if $i \in \overline{I_{C}}, x_{i} \geq 0$ otherwise, and $\sum_{i} x_{i} \leq 1$. Clearly, one can see that this last inequality is an equality when $I_{C} \neq \emptyset$ (otherwise $K$ is not minimum).

To minimize the function, we compute the partial derivatives of $K$ :

$$
\forall i \in I_{C}, \frac{\partial K(\mathcal{X})}{\partial x_{i}}=-\alpha \frac{w_{i} f_{i} d_{i}}{x_{i}^{\alpha+1}} .
$$

By setting them all to 0 , we obtain the following equality for $1 \leq i \leq n$ :

$$
-\alpha \frac{w_{i} f_{i} d_{i}}{x_{i}^{\alpha+1}}=-\alpha \frac{w_{n} f_{n} d_{n}}{x_{n}^{\alpha+1}}
$$

Hence,

$$
\begin{aligned}
\forall i \in I_{C}, x_{i} & =x_{n} \frac{\left(w_{i} f_{i} d_{i}\right)^{\frac{1}{\alpha+1}}}{\left(w_{n} f_{n} d_{n}\right)^{\frac{1}{\alpha+1}}} \\
\sum_{i=1}^{n} x_{i} & =\frac{x_{n}}{\left(w_{n} f_{n} d_{n}\right)^{\frac{1}{\alpha+1}}} \sum_{i \in I_{C}}\left(w_{i} f_{i} d_{i}\right)^{\frac{1}{\alpha+1}} \\
& =1 .
\end{aligned}
$$

Hence, the desired result.

Definition 4 (Dominant partition). Given a set of applications $T_{1}, \ldots, T_{n}$, we say that a partition of these applications $I_{C}, \overline{I_{C}}$ is dominant, if for all $i \in I_{C}$,

$$
\frac{\left(w_{i} f_{i} d_{i}\right)^{1 /(\alpha+1)}}{\sum_{j \in I_{C}}\left(w_{j} f_{j} d_{j}\right)^{1 /(\alpha+1)}}>d_{i}^{1 / \alpha} .
$$

We can now state the following result:

Theorem 2. If a partition $I_{C}, \overline{I_{C}}$ is not dominant, then we can compute in polynomial time a better solution.

Proof. Let $I_{C}, \overline{I_{C}}$ be a non-dominant partition.

Let $i_{0} \in I_{C}$ such that $\frac{\left(w_{i_{0}} f_{i_{0}} d_{i_{0}}\right)^{1 /(\alpha+1)}}{\sum_{j \in I_{C}}\left(w_{j} f_{j} d_{j}\right)^{1 /(\alpha+1)}} \leq d_{i_{0}}^{1 / \alpha}$.

First we can show that there is $i_{1} \in I_{C} \backslash\left\{i_{0}\right\}$. Indeed, otherwise we would have $\frac{\left(w_{i_{0}} f_{i_{0}} d_{i_{0}}\right)^{1 /(\alpha+1)}}{\sum_{j \in I_{C}}\left(w_{j} f_{j} d_{j}\right)^{1 /(\alpha+1)}}=$ 
$1 \leq d_{i_{0}}^{1 / \alpha}$, and $I_{C}, \overline{I_{C}}$ is not a valid partition: then COSCHEDCACHE-PART $\left(I_{C}, \overline{I_{C}}\right)$ does not admit any solution.

Let $\mathcal{T}_{e}$ (resp. $\mathcal{T}_{p}$ ) be the optimal execution time of COSCHEDCACHE-Ext $\left(I_{C}, \overline{I_{C}}\right)$ (resp. $\operatorname{CoSchedCACHE-PaRt}\left(I_{C}, \overline{I_{C}}\right)$ ). We know that $\mathcal{T}_{e} \leq \mathcal{T}_{p}$. Let us further denote by $\mathcal{X}=\left\{x_{1}, \ldots, x_{n}\right\}$ the optimal solution to CoSCHEDCACHE-EXT $\left(I_{C}, \overline{I_{C}}\right)$. Let $\overline{\mathcal{X}}=\left\{\bar{x}_{1}, \ldots, \bar{x}_{n}\right\}$ be such that (i) $\bar{x}_{i_{0}}=0$, (ii) $\bar{x}_{i_{1}}=x_{i_{0}}+x_{i_{1}}$, and (iii) $\bar{x}_{i}=x_{i}$ for all other $i$ 's.

Then clearly $\overline{\mathcal{X}}$ is a solution, and we have:

$$
\begin{array}{cl}
\mathcal{E x e}_{i_{0}}^{\text {seq }}\left(\bar{x}_{i_{0}}\right) \leq w_{i_{0}}\left(1+f_{i_{0}} l_{s}+f_{i_{0}} l_{l} \frac{d_{i_{0}}}{x_{i_{0}}^{\alpha}}\right) ; & \\
\mathcal{E x e}_{i_{1}}^{\text {seq }}\left(\bar{x}_{i_{1}}\right)<w_{i_{1}}\left(1+f_{i_{1}} l_{s}+f_{i_{1}} l_{l} \frac{d_{i_{0}}}{x_{i_{1}}^{\alpha}}\right) ; & \\
\mathcal{E x e}_{i}^{\text {seq }}\left(\bar{x}_{i}\right) \leq w_{i}\left(1+f_{i} l_{s}+f_{i} l_{l} \frac{d_{i}}{x_{i}^{\alpha}}\right) & \text { if } i \in I_{C} ; \\
\mathcal{E x e}_{i}^{\text {seq }}\left(\bar{x}_{i}\right)=w_{i}\left(1+f_{i}\left(l_{s}+l_{l}\right)\right) & \text { if } i \in \overline{I_{C}} .
\end{array}
$$

Indeed, these results are direct consequences of the definition of $\mathcal{E} x \mathrm{e}^{\text {seq }}$, except Equation (5), which we establish as follows:

- If $x_{i_{1}} \geq d_{i_{1}}^{1 / \alpha}$, then $\bar{x}_{i_{1}}>d_{i_{1}}^{1 / \alpha}$

$$
\begin{aligned}
\mathcal{E x e}_{i_{1}}^{\mathrm{seq}}\left(\bar{x}_{i_{1}}\right) & =w_{i_{1}}\left(1+f_{i_{1}} l_{s}+f_{i_{1}} l_{l} \frac{d_{i_{0}}}{\bar{x}_{i_{1}}^{\alpha}}\right) \\
& <w_{i_{1}}\left(1+f_{i_{1}} l_{s}+f_{i_{1}} l_{l} \frac{d_{i_{0}}}{x_{i_{1}}^{\alpha}}\right) .
\end{aligned}
$$

- If $x_{i_{1}}<d_{i_{1}}^{1 / \alpha}$, then for all $x \in[0,1], \mathcal{E x e}_{i_{1}}^{\mathrm{seq}}(x)<$ $w_{i_{1}}\left(1+f_{i_{1}} l_{s}+f_{i_{1}} l_{l} \frac{d_{i_{0}}}{x_{i_{1}}^{\alpha}}\right)$.

Hence:

$$
\begin{gathered}
\frac{1}{p} \sum_{i=1}^{n} \mathcal{E x e}_{i}^{\mathrm{seq}}\left(\bar{x}_{i}\right)<\frac{1}{p}\left(\sum_{i \in \overline{I_{C}}} w_{i}\left(1+f_{i}\left(l_{s}+l_{l}\right)\right)\right. \\
\left.+\sum_{i \in I_{C}} w_{i}\left(1+f_{i} l_{s}+f_{i} l_{l} \frac{d_{i}}{x_{i}^{\alpha}}\right)\right)=\mathcal{T}_{e} \leq \mathcal{T}_{p}
\end{gathered}
$$

which shows that $\overline{\mathcal{X}}$ is a better solution computed in polynomial time from $\mathcal{X}$. Furthermore, by construction of $\overline{\mathcal{X}}$, we have strictly decreased the size of the new set $I_{C}$.

Finally, we can show a second dominance result:

Theorem 3. If a partition $I_{C}, \overline{I_{C}}$ is dominant, then the optimal solution to COSCHEDCACHE-PART $\left(I_{C}, \overline{I_{C}}\right)$ is:

$$
\begin{array}{ll}
x_{i}=\frac{\left(w_{i} f_{i} d_{i}\right)^{1 /(\alpha+1)}}{\sum_{j \in I_{C}}\left(w_{j} f_{j} d_{j}\right)^{1 /(\alpha+1)}} & \text { if } i \in I_{C} ; \\
x_{i}=0 & \text { otherwise. }
\end{array}
$$

Proof. This is a corollary of Lemma 4, this solution is the optimal solution to COSCHEDCACHE-EXT $\left(I_{C}, \overline{I_{C}}\right)$ and it is a valid solution to COSCHEDCACHE-PART $\left(I_{C}, \overline{I_{C}}\right)$, hence it is the optimal solution to CoSChedCACHE-PART $\left(I_{C}, \overline{I_{C}}\right)$.

\section{HEURISTICS}

In this section, we aim at designing efficient heuristics for general applications that obey Amdahl's law, and whose memory footprints are larger than the cache size $\left(a_{i}=+\infty\right)$. However, the COSCHEDCACHE problem seems to be very difficult for such applications. In particular, Lemma 2 does not hold, and we have no guideline to decide how many processors to assign. We simplify the design of the heuristics by temporarily allocating processors as if the applications were perfectly parallel, and then concentrating on strategies that partition the cache efficiently among some applications (and give no cache fraction to remaining ones). In accordance with Theorem 2, our goal is to compute dominant partitions. Recall that $I_{C}$ represents the subset of applications that receive a fraction fo the cache. Once a dominant partition is given, we obtain the schedule $\mathcal{S}=\left\{\left(x_{i}, p_{i}\right)\right\}_{i}$ as follows: first we determine the $x_{i}$ 's with Theorem 3, and then we recompute the $p_{i}$ 's so that all applications complete simultaneously at time $K$. However, there is no longer a nice analytical characterization of the makespan $K$, hence we use a binary search to compute $K$ as follows: for each application $T_{i}$, the execution time writes $\left(s_{i}+\frac{1-s_{i}}{p_{i}}\right) c_{i}=K$, where $s_{i}$ is the sequential fraction, and $c_{i}=w_{i}\left(1+f_{i}\left(l_{s}+l_{l} \frac{d_{i}}{x_{i}^{\alpha}}\right)\right)$ if $T_{i} \in I_{C}$, or $c_{i}=w_{i}\left(1+f_{i}\left(l_{s}+l_{l}\right)\right)$ otherwise. From $\sum_{i=1}^{x_{i}} p_{i}=p$, we derive the equation

$$
\sum_{i=1}^{n} \frac{1-s_{i}}{\frac{K}{c_{i}}-s_{i}}=p
$$

and we compute $K$ through a binary search. A lower (resp. upper) bound for $K$ is to assign $p$ (resp. 1) processor(s) to each application.

To compute dominant partitions, we use two greedy strategies:

- Dominant: we start with $I_{C}=\mathcal{I}$ and greedily remove some applications from $I_{C}$ until we have a dominant partition (see Algorithm 1);

- DominantRev: initially $I_{C}$ is empty, and we greedily add applications while $I_{C}$ remains dominant (see Algorithm 2).

In both strategies, the greedy criterion to select the next application is the choice function taken from the following three alternatives:

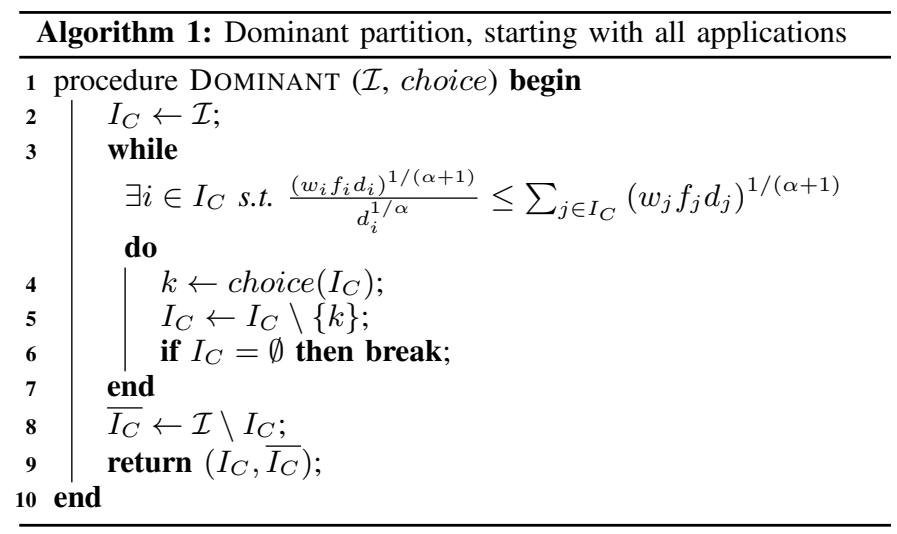




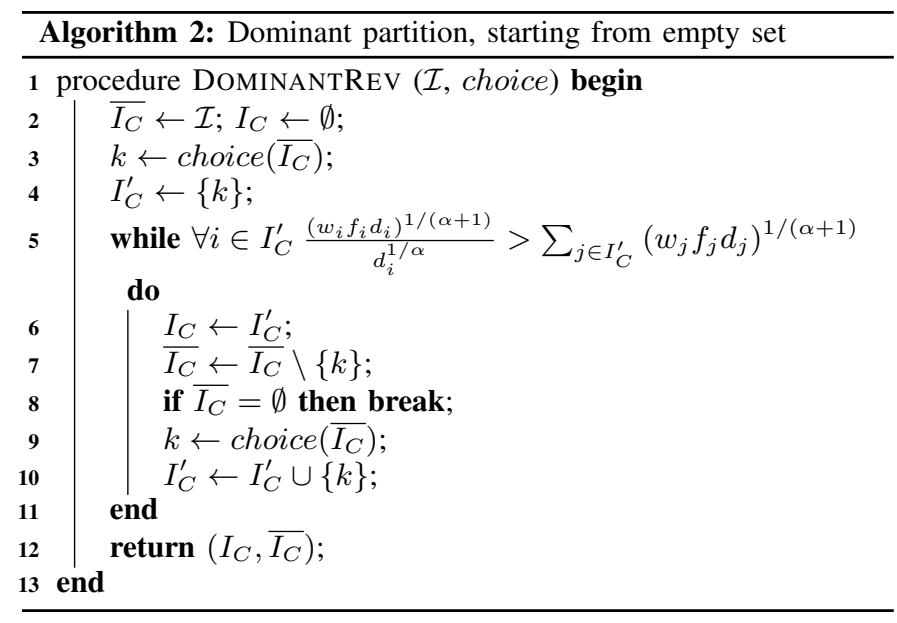

- RANDOM: choice $(\mathcal{I})$ picks up randomly one application among all applications;

- MinRatio considers the ratio that appears in Definition 4 (dominant partitions), and chooses an application with a small ratio:

$$
\operatorname{choice}(\mathcal{I})=\arg \min _{i \in \mathcal{I}}\left(\frac{\left(w_{i} f_{i} d_{i}\right)^{1 /(\alpha+1)}}{d_{i}^{1 / \alpha}}\right) ;
$$

- MAXRATio proceeds the other way round, by choosing an application with a large ratio:

$$
\operatorname{choice}(\mathcal{I})=\arg \max _{i \in \mathcal{I}}\left(\frac{\left(w_{i} f_{i} d_{i}\right)^{1 /(\alpha+1)}}{d_{i}^{1 / \alpha}}\right) \text {. }
$$

The intuition behind these heuristics is the following: applications that render a solution non dominant are such that (see Definition 4):

$$
\frac{\left(w_{i} f_{i} d_{i}\right)^{1 /(\alpha+1)}}{d_{i}^{1 / \alpha}} \leq \sum_{j \in I_{C}}\left(w_{j} f_{j} d_{j}\right)^{1 /(\alpha+1)} .
$$

Hence, we expect to reach dominance faster by removing from a non-dominant solution applications with low $\frac{\left(w_{i} f_{i} d_{i}\right)^{1 /(\alpha+1)}}{d_{i}^{1 / \alpha}}$ (left term of the equation). Intuitively, DoMINANT should work well with the MinRATIO criterion. For symmetric reasons, we expect DOMINANTREV to work well with the MAXRATIO criterion. These intuitions will be experimentally confirmed in Section VI. Altogether, by combining two strategies and three different choice functions, we obtain six heuristics to build dominant partitions.

\section{Simulations}

To assess the efficiency of the heuristics defined in Section $\mathrm{V}$, we have performed extensive simulations. The simulation settings are discussed in Section VI-A, and results are presented in Sections VI-B and VI-C. The code is publicly available at http://perso.ens-lyon.fr/loic.pottier/archives/ simu-cache.zip.

\section{A. Simulation settings}

We use data from applicative benchmarks to run the experiments. Table I provides a brief description of the NAS Parallel Benchmark (NPB) suite [4], and Table II shows the

\begin{tabular}{ll} 
App & Description \\
\hline CG & $\begin{array}{l}\text { Uses conjugate gradients method to solve a large } \\
\text { sparse symmetric positive definite system of linear } \\
\text { equations }\end{array}$ \\
\hline BT & $\begin{array}{l}\text { Solves multiple, independent systems of block } \\
\text { tridiagonal equations with a predefined block size }\end{array}$ \\
\hline LU & $\begin{array}{l}\text { Solves regular sparse upper and lower triangular } \\
\text { systems }\end{array}$ \\
\hline SP & $\begin{array}{l}\text { Solves multiple, independent systems of scalar } \\
\text { pentadiagonal equations }\end{array}$ \\
\hline MG & $\begin{array}{l}\text { Performs a multi-grid solve on a sequence of } \\
\text { meshes }\end{array}$ \\
\hline FT & Performs discrete 3D fast Fourier Transform \\
\hline
\end{tabular}

Table I: Description of the NPB benchmarks.

\begin{tabular}{rccc} 
App & $w_{i}$ & $f_{i}$ & $m_{40 \mathrm{MB} \mathcal{S}_{s}}^{i}$ \\
\hline CG & $5.70 \mathrm{E}+10$ & $5.35 \mathrm{E}-01$ & $6.59 \mathrm{E}-04$ \\
BT & $2.10 \mathrm{E}+11$ & $8.29 \mathrm{E}-01$ & $7.31 \mathrm{E}-03$ \\
LU & $1.52 \mathrm{E}+11$ & $7.50 \mathrm{E}-01$ & $1.51 \mathrm{E}-03$ \\
SP & $1.38 \mathrm{E}+11$ & $7.62 \mathrm{E}-01$ & $1.51 \mathrm{E}-02$ \\
MG & $1.23 \mathrm{E}+10$ & $5.40 \mathrm{E}-01$ & $2.62 \mathrm{E}-02$ \\
FT & $1.65 \mathrm{E}+10$ & $5.82 \mathrm{E}-01$ & $1.78 \mathrm{E}-02$
\end{tabular}

Table II: Experimental values from NPB benchmarks.

parameters for these six HPC applications. We obtain the values shown in Table II by instrumenting and simulating the benchmarks $(C L A S S=A)$ on 16 cores using PEBIL [19]. For the simulations, we use a cache configuration representing an Intel Xeon CPU E5-2690, with a 20MB last level cache per processor of 8 cores. Since the cache miss ratio is defined for a $40 \mathrm{MB}$ cache, we have $d_{i}=m_{40 \mathrm{MBS}_{s}}\left(\frac{40 \times 10^{6}}{C_{s}}\right)^{\alpha}$.

We build synthetic applications from Table II by varying the work $w_{i}$ randomly between $1 \mathrm{E}+8$ and $1 \mathrm{E}+12$. Other data sets building upon these applications have been used (see the companion research report [3]), and the results are very similar. The sequential fraction of work $s_{i}$ is taken randomly between $1 \%$ and $15 \%$.

For the execution platform, we consider one manycore Sunway TaihuLight [8] with 256 processors and a shared memory of 32GB. We chose this platform because of its high core count. Strictly speaking, this platform does not have a last level cache (LLC), but the shared memory can be seen as the LLC, using the disk as the large memory. We have $C_{s}=32000 \times 10^{6}$. The large storage latency $l_{l}$ is set to 1 . The small storage latency $l_{s}$ is set to 0.17 . According to the literature [18], [22], [24], the last level cache (LLC) latency is on average four to ten times better than the DDR latency, and we enforce a ratio of 5.88 in the simulations. We have used different ratios in [3], and they lead to similar results. Finally, the Power Law parameter is fixed to $\alpha=0.5$. We execute each heuristic 50 times and we compute the average makespan, i.e., the longest execution time among all coscheduled applications.

In Section VI-B, we provide a comparison of the six heuristics of Section V, before assessing the gain due to coscheduling in Section VI-C.

\section{B. Comparison of the six heuristics}

Figure 1 shows the normalized makespan obtained by the six variants of heuristics building dominant partitions. Results 


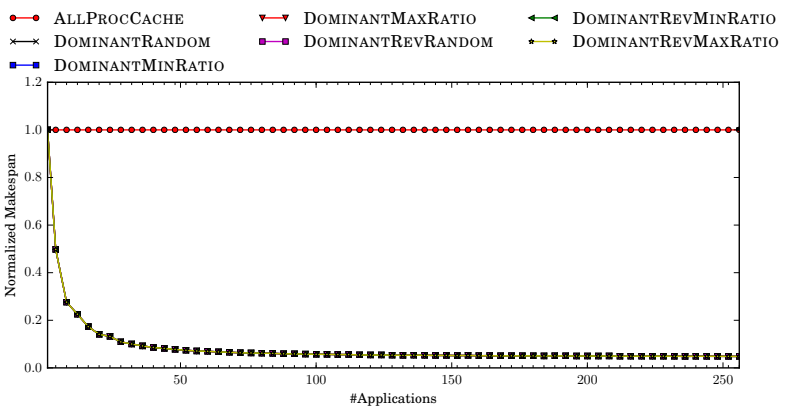

Figure 1: Comparison of the six dominant partition heuristics.

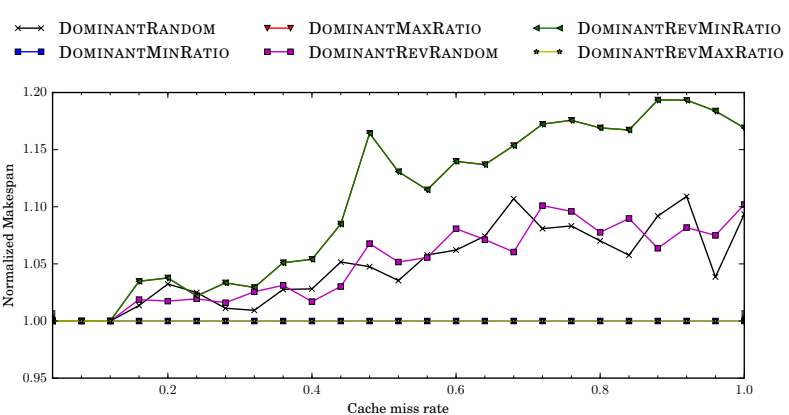

Figure 2: Impact of cache miss rate using a 1GB LLC.

are normalized with the makespan of ALLPROCCACHE, which is the execution without any co-scheduling: in the ALLPROCCACHE heuristic, applications are executed sequentially, each using all processors and all the cache. We vary the number of applications from 1 to 256 on 256 processors. The six heuristics obtain similarly good results, with a gain of $85 \%$ over AlLPROCCACHE as soon as there are at least 50 applications.

Since all six variants show the same performance on the previous data sets, we investigate the impact of the cache miss rate by varying it between 0 and 1 with a LLC of $C_{s}=1 G B$ in Figure 2. Results are now normalized with DOMINANTMinRatio, which enables to zoom out the differences. Actually, DominantMinRatio and DominantRevMaxRATIO are always the best (their plots overlap), while DOMINANTMAXRATIO and DOMINANTREVMINRATIO are almost always the worst (again, their plots overlap), hence confirming the intuition in Section V. The random variants lay in between. We use a 1GB LLC to show the impact of cache miss rate on heuristic behaviors. We observe that there are some differences between heuristics only when the cache miss ratio becomes greater than 0.1 . According to current data, $m_{40 \mathrm{MB} \mathcal{S}_{s}}$ range from $1 \mathrm{E}-02$ to $1 \mathrm{E}-04$. In addition, these differences are visible only with a small shared memory (1GB in the example) while our execution platform has a 32GB shared memory. Overall, for the system used in these simulations, all heuristics perform similarly, even though DOMINANTMINRATIO and DOMINANTREVMAXRATIO seem to perform best in all other settings that we tried (see [3]). Therefore, for clarity, we plot only one heuristic based on dominant partitions in the remaining simulations, namely DOMINANTMINRATIO.

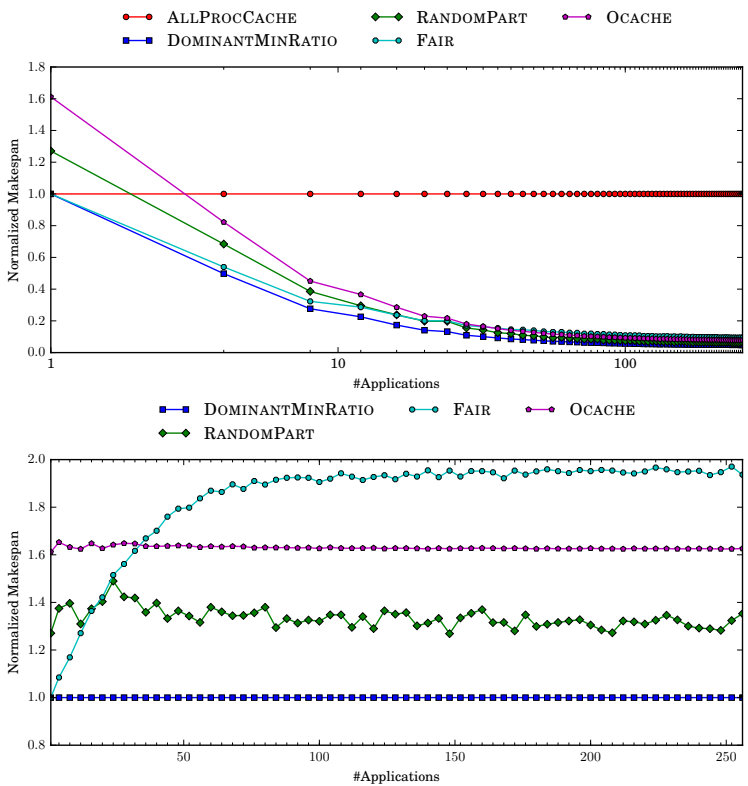

Figure 3: Impact of the number of applications.

\section{Gain with co-scheduling}

In this section, we assess the gain due to co-scheduling by comparing DominAnTMInRATIO with AllPROCCACHE and with three other heuristics:

- FAIR gives $p_{i}=\frac{p}{n}$ processors, and a fraction of cache $x_{i}=$ $\frac{f_{i}}{\sum_{j=1}^{n} f_{j}}$ to each application;

- OCACHE gives no cache to any application, i.e., $x_{i}=0$ for $1 \leq i \leq n$, and then it computes the $p_{i}$ 's so that all applications finish at the same time;

- RANDOMPART randomly partitions applications with and without cache. For those in cache, the $x_{i}$ 's are computed with the method used for dominant partitions. Then, the $p_{i}$ 's are computed so that all applications finish at the same time.

Impact of the number of applications. Figure 3 (normalized with AllPRocCACHE) shows the impact of the number of applications when the number of processors is fixed to 256 . We see that DominANTMINRATIO outperforms the other heuristics, hence showing the efficiency of our approach based on dominant partitions. Results are also normalized with DOMINANTMINRATIO, so that we can better observe the differences between co-scheduling heuristics. FAIR exhibits good results only for a small number of applications, when all applications can fit into cache. Otherwise, the use of dominant partitions is much more efficient, as seen with RANDOMPART, or even 0CACHE that does not use cache but ensures that all applications finish at the same time.

Figure 4 shows the impact of the average number of processors per application. Here, results are normalized with DOMINANTMINRATIO again, and they are similar. In particular, we see that 0CACHE is better than FAIR when there are many processors to share between applications, even though it is not using the cache at all. These results show the accuracy of the model and the benefits of using dominant partitions. 


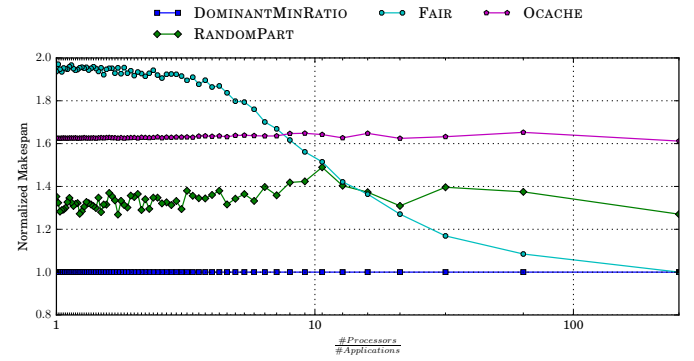

Figure 4: Impact of the average number of processors per applications.

Finally, we note the importance of cache partitioning, since the difference between 0CACHE and DOMINANTMINRATIO relies on cache allocation.

Impact of the number of processors. Figure 5 (normalized with AlLPROCCACHE) shows the impact of the number of processors when the number of applications is fixed to 16 . When the number of processors increases, the gain of coscheduling increases. DOMINANTMINRATIO is clearly the best heuristic. RANDOMPART, which builds a random partition instead of a dominant one, is outperformed by DOMINANTMinRATIO, and the latter is the only heuristic that surpasses AlLPROCCACHE when the number of processors is low. So, building a dominant partition seems a good strategy to optimize the makespan.

The normalization with DominantMinRatio shows that when the number of processors increases, FAIR becomes better, while RANDOMPART and 0CACHE are quite stable since they are based on the same model as DominantMinRatio. The only difference between 0CACHE and DOMINANTMINRATIO is the cache allocation strategy, and the gain from cleverly distributing cache fractions across applications exceeds $20 \%$. With more applications, we obtain the same ranking of heuristics, except that FAIR is always the worst heuristic: since there are less processors on average per application, a good co-scheduling policy is necessary (see [3] for detailed results).

Impact of the sequential fraction of work. Figure 6 (normalized with ALLPROCCACHE) shows the impact of the sequential part $s_{i}$ when the number of processors is fixed to 256. The number of applications is fixed to 16. As expected, when the sequential fraction of work increases, all coscheduling heuristics perform better than ALLPROCCACHE, and DominantMinRatio is always the best heuristic. It leads to a gain of more than $50 \%$ when $s_{i}=0.01$.

The normalization with DOMINANTMINRATIO better shows the impact of the sequential part: we observe that when the sequential fraction of work increases, FAIR obtains results closer to DominantMinRatio.

Summary. To summarize, all heuristics based on dominant partitions are very efficient, especially when compared to the classical heuristics FAIR (which share the cache fairly between applications) and ALLPROCCACHE (which does no co-scheduling). The unexpected result that can be observed is that the gain brought by our heuristics comes even with

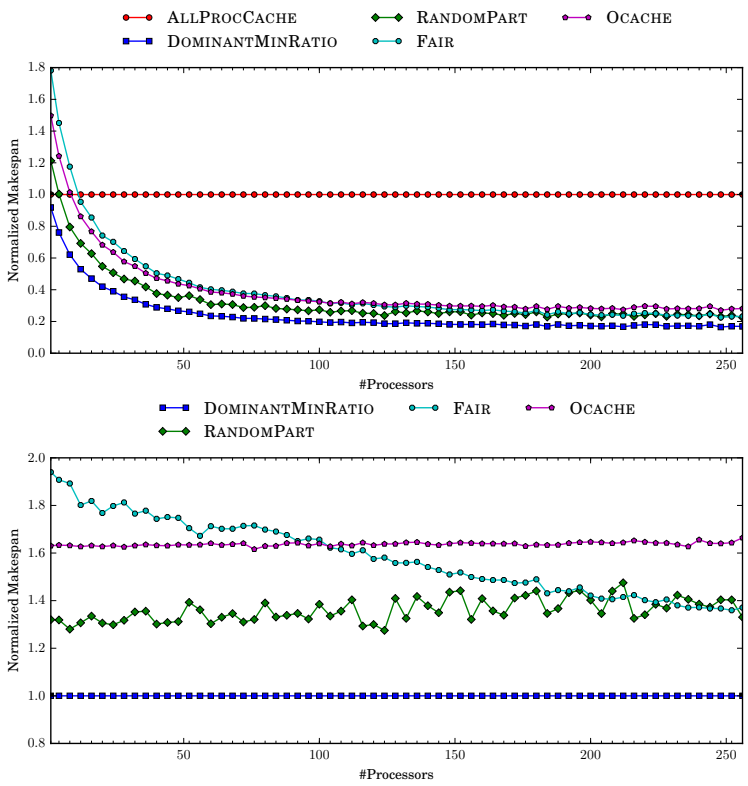

Figure 5: Impact of the number of processors.

$\because$ allProccache $\because$ RandomPart $\because$ Ocache $\because$ DominantMinRatio $\because$ Fair

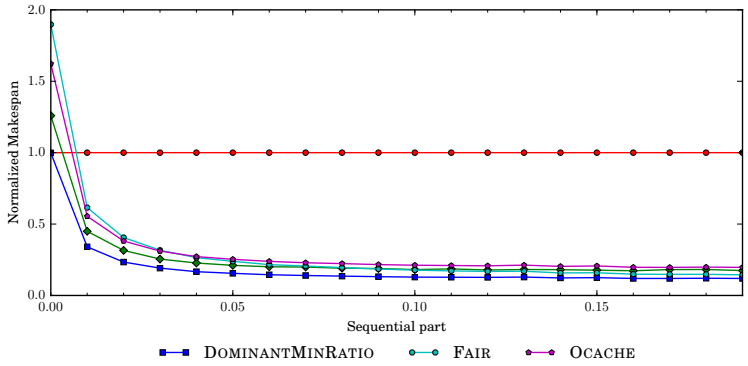
$\leftrightarrow$ RandomPart

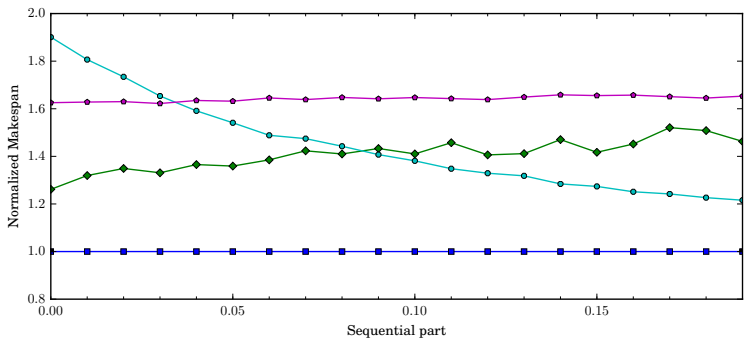

Figure 6: Impact of sequential fraction of work.

very low sequential time (below 0.01)! This is unexpected since the natural intuition would be a behavior such as the one observed on FAIR: a makespan up to 1.9 times longer than AllProcCache with low sequential time.

We show that the ratio processors/applications has a significant impact on performance: when many processors are available for a few applications, it is less crucial to use efficient cache-partitioning and all applications can share the cache, hence FAIR obtains good results, close to DOMINANTMINRATIO. Otherwise, RANDOMPART is the second best heuristic, followed by 0CACHE that does not use the cache, then FAIR and AllProcCache.

All heuristics run within a very small time (less than ten seconds in the worst of the settings used, to be compared with 
a typical application execution time in hours or days), hence they can be used in practice with a very light overhead.

\section{CONCLUSION}

In this paper, we have provided a preliminary study on coscheduling algorithms for cache-partitioned systems, building upon a theoretical study for perfectly parallel applications. The two key scheduling questions are (i) which proportion of cache and (ii) how many processors should be given to each application. We proved that the problem is NP-complete, but we have been able to characterize optimal solutions for perfectly parallel applications by introducing the concept of dominant partitions: for such applications, we have computed the optimal proportion of cache to give to each application in the partition. Furthermore, we have provided explicit formulas to express the number of processors to assign to each application.

Several polynomial-time heuristics have been built upon these results, and extensive simulation results demonstrated that the use of dominant partitions always leads to better results than more naive approaches, as soon as there is a small sequential fraction of work in application speedup profiles. The concept of sharing the cache only between a subset of applications seems highly relevant, since even an approach with a random selection of applications that share the cache leads to good results. Also, a clever partitioning of the cache pays off quite well, since our heuristics lead to a significant gain compared to an approach where no cache is given to applications. Overall, the heuristics appear to be very useful for general applications, even though their cache allocation strategy relies on simulating a perfectly parallel profile.

Future work will be devoted to extending the heuristics that account for the speedup profile for both processor and cache allocation. Also, we would like to gain access to, and conduct real experiments on, a cache-partitioned system with a high core count: this would allow us to further validate the accuracy of the model and to confirm the impact of our promising results.

\section{REFERENCES}

[1] Advanced Scientific Computing Advisory Committee (ASCAC), "Ten technical approaches to address the challenges of Exascale computing."

[2] G. Amdahl, "The validity of the single processor approach to achieving large scale computing capabilities," in AFIPS Conference Proceedings, vol. 30. AFIPS Press, 1967, pp. 483-485.

[3] G. Aupy, A. Benoit, L. Pottier, P. Raghavan, Y. Robert, and M. Shantharam, "Co-scheduling algorithms for cache-partitioned systems," INRIA, Research report RR-8965, 2016, at graal.ens-lyon.fr/ abenoit.

[4] D. H. Bailey et al., "The NAS Parallel Benchmarks - Summary and Preliminary Results," in Proc. SC'91. ACM, 1991, pp. 158-165.

[5] S. Blagodurov, S. Zhuravlev, and A. Fedorova, "Contention-aware scheduling on multicore systems," ACM Trans. Comput. Syst., vol. 28, no. 4, pp. 8:1-8:45, 2010.

[6] B. D. Bui, M. Caccamo, L. Sha, and J. Martinez, "Impact of cache partitioning on multi-tasking real time embedded systems," in Embedded and Real-Time Computing Syst.and App., 2008, pp. 101-110.

[7] D. Dauwe, E. Jonardi, R. Friese, S. Pasricha, A. A. Maciejewski, D. A. Bader, and H. J. Siegel, "A methodology for co-location aware application performance modeling in multicore computing," in IPDPS Workshops, 2015, pp. 434-443.
[8] J. Dongarra, "Report on the sunway taihulight system," $P D F)$. www. netlib. org. Retrieved June, vol. 20, 2016.

[9] T. Dwyer, A. Fedorova, S. Blagodurov, M. Roth, F. Gaud, and J. Pei, "A practical method for estimating performance degradation on multicore processors, and its application to hpc workloads," in Proc. SC'12, 2012, pp. 83:1-83:11.

[10] A. Gainaru, G. Aupy, A. Benoit, F. Cappello, Y. Robert, and M. Snir, "Scheduling the I/O of HPC applications under congestion," in IEEE IPDPS, 2015, pp. 1013-1022.

[11] M. R. Garey and D. S. Johnson, Computers and Intractability, a Guide to the Theory of NP-Completeness. W.H. Freeman and Company, 1979.

[12] N. Guan, M. Stigge, W. Yi, and G. Yu, "Cache-aware scheduling and analysis for multicores," in Proc. 7th ACM Int. Conf. Embedded Software, ser. EMSOFT '09. ACM, 2009, pp. 245-254.

[13] A. Hartstein, V. Srinivasan, T. Puzak, and P. Emma, "On the nature of cache miss behavior: Is it $\sqrt{2}$," The Journal of Instruction-Level Parallelism, vol. 10, pp. 1-22, 2008.

[14] L. He, H. Zhu, and S. A. Jarvis, "Developing graph-based co-scheduling algorithms on multicore computers," IEEE Trans. Parallel Distributed Systems, vol. 27, no. 6, pp. 1617-1632, 2016.

[15] Intel, "Intel 64 and IA-32 architectures software developer's manual," Part 2, vol. 3B: System Programming Guide, 2014.

[16] Y. Jiang, X. Shen, J. Chen, and R. Tripathi, "Analysis and approximation of optimal co-scheduling on chip multiprocessors," in Proc. Parallel Architectures Compilation Techniques, ser. PACT, 2008, pp. 220-229.

[17] A. Krishna, A. Samih, and Y. Solihin, "Data sharing in multi-threaded applications and its impact on chip design," in Int. Symp. Performance Analysis of Systems and Software (ISPASS). IEEE, 2012, pp. 125-134.

[18] E. Kultursay, M. Kandemir, A. Sivasubramaniam, and O. Mutlu, "Evaluating STT-RAM as an energy-efficient main memory alternative," in IEEE ISPASS, 2013, pp. 256-267.

[19] M. A. Laurenzano, M. M. Tikir, L. Carrington, and A. Snavely, "PEBIL: Efficient static binary instrumentation for Linux," in IEEE ISPASS, 2010, pp. 175-183.

[20] J. Leverich and C. Kozyrakis, "Reconciling high server utilization and sub-millisecond quality-of-service," in Proceedings of the Ninth European Conference on Computer Systems. ACM, 2014, p. 4.

[21] D. Lo, L. Cheng, R. Govindaraju, P. Ranganathan, and C. Kozyrakis, "Improving resource efficiency at scale with Heracles," ACM Transactions on Computer Systems (TOCS), vol. 34, no. 2, p. 6, 2016.

[22] D. Molka, D. Hackenberg, R. Schone, and W. E. Nagel, "Cache Coherence Protocol and Memory Performance of the Intel Haswell-EP Architecture," in ICPP, Sept 2015, pp. 739-748.

[23] S. P. Muralidhara, L. Subramanian, O. Mutlu, M. Kandemir, and T. Moscibroda, "Reducing memory interference in multicore systems via application-aware memory channel partitioning," in Proc. MICRO. ACM, 2011, pp. 374-385.

[24] A. J. Pena and P. Balaji, "Toward the efficient use of multiple explicitly managed memory subsystems," in IEEE CLUSTER, 2014, pp. 123-131.

[25] M. K. Qureshi and Y. N. Patt, "Utility-based cache partitioning: A low-overhead, high-performance, runtime mechanism to partition shared caches," in Proc. MICRO, 2006, pp. 423-432.

[26] B. M. Rogers, A. Krishna, G. B. Bell, K. Vu, X. Jiang, and Y. Solihin, "Scaling the bandwidth wall: challenges in and avenues for CMP scaling," in ACM SIGARCH Computer Architecture News, vol. 37, no. 3. ACM, 2009, pp. 371-382.

[27] C. Sewell, K. Heitmann, H. Finkel, G. Zagaris, S. T. Parete-Koon, P. K Fasel, A. Pope, N. Frontiere, L.-t. Lo, B. Messer et al., "Large-scale compute-intensive analysis via a combined in-situ and co-scheduling workflow approach," in Proc. SC'15. ACM, 2015, p. 50.

[28] K. Tian, Y. Jiang, and X. Shen, "A study on optimally co-scheduling jobs of different lengths on chip multiprocessors," in Proc. 6th ACM Conf. Computing Frontiers, ser. CF '09. ACM, 2009, pp. 41-50.

[29] Y. Zhang, M. A. Laurenzano, J. Mars, and L. Tang, "Smite: Precise QOSprediction on real-system SMT processors to improve utilization in warehouse scale computers," in Proc. MICRO, 2014, pp. 406-418.

[30] H. Zhu, L. He, B. Gao, K. Li, J. Sun, H. Chen, and K. Li, "Modelling and developing co-scheduling strategies on multicore processors," in Proc. ICPP, 2015, pp. 220-229.

[31] S. Zhuravlev, S. Blagodurov, and A. Fedorova, "Addressing shared resource contention in multicore processors via scheduling," in $A C M$ Sigplan Notices, vol. 45, no. 3. ACM, 2010, pp. 129-142. 